\title{
Reconstructive Surgery in the Lower Urinary Tract in Children: Can the Complication Rate Be Reduced?
}

\author{
Sara Carlsson, Maryam Moussavi, Ulla Sillen, Gundela Holmdahl, Kate Abrahamsson* \\ Pediatric Urology Section, Pediatric Surgery Department, The Queen Silvia Children's Hospital, Sahlgrenska \\ University Hospital, Gothenburg, Sweden \\ Email: ${ }^{*}$ Kate.Abrahamsson@vgregion.se
}

Received 22 January 2014; revised 22 February 2014; accepted 28 February 2014

Copyright (C) 2014 by authors and Scientific Research Publishing Inc.

This work is licensed under the Creative Commons Attribution International License (CC BY). http://creativecommons.org/licenses/by/4.0/

\section{Open Access}

\begin{abstract}
Objective: Reconstructive surgery of the lower urinary tract in children is reported with a high complication rate. The aim was to evaluate the complication rate at our institution. Material and methods: Between 2000 and 2010, 41 boys and 19 girls were consecutively operated on with augmentation with ileum (45), alternative CIC-channel (57) and bladder neck plasty (42) in isolation or as combined procedures in individuals with neurogenic bladder dysfunction NBD (42), bladder exstrophy-epispadias complex BEEC (13), isolated epispadias IE (2) and posterior urethral valves, PUV (3). Median age at surgery was 11 years (range 1.3 - 21) and median follow-up time 7 years (1 10). Complications were consecutively observed at follow-up according to a structured protocol. As first line care, specially trained nurses followed the patients and daily bladder irrigation was included in the CIC follow-up regimen. Results: In individuals with augmentation with ileum, of which all but one performed CIC through an alternative channel, there were stones reported in $3 / 45(7 \%)$, perforation in $2 / 45(4 \%)$, reoperation of CIC channel in 5/57 (9\%), bowel obstruction in 3/56 (5\%) and rupture of BNP in 3/39 (8\%). Re-augmentation was not needed and malignancy not found. No significant difference was seen between patients with NBD and BEEC/IE. Conclusion: Complication rates were among the lowest reported for stones, perforation and reoperations of CIC channels and were average for bowel obstruction. Bladder stones and perforation were seen in individuals with bad compliance to recommended CIC- and irrigation regimens.
\end{abstract}

\section{Keywords}

Children; Complications; Augmentation; CIC-Channel; Bladder Neck Plasty

"Corresponding author. 


\section{Introduction}

In spite of anticholinergic treatment in care of the low compliant urinary bladder, reconstructive surgery is still needed in some children to create a low pressure reservoir. In addition, there is often a need for enhancing continence due to an insufficient urethral sphincter. Since clean intermittent catheterization (CIC) can be performed through an alternative catheterizable channel, bladder neck plasty (BNP) can be used to make a neourethra both tight and long. If such a procedure is done without augmentation a common complication of an increased outlet resistance is untreatable bladder overactivity in the native bladder. Unfortunately it is not possible to predict which bladders will develop such overactivity [1]. Therefore a common procedure in patients with NBD is to augment the bladder simultaneously.

Many reports present high incidence of complications following reconstructive surgery in the lower urinary tracts [2] [3]. The high complication rate might partly be due to additional problems associated with the patients in question. Patients needing this intervention are children with neurogenic bladder dysfunction (NBD) often due to myelomeningocele (MMC), and children with malformations in the lower urinary tract, mainly due to bladder exstrophy-epispadias complex (BEEC). In MMC children, cognitive disturbances are often seen, and in BEEC puberty is the challenging period for care of the reservoir.

The aim of this study was to evaluate the complication rate after reconstructive surgery at our department. In addition, try to identify predictive factors for the development of complications.

\section{Patients and Methods}

All children with MMC living in western Sweden with a total population of approximately 2.5 million, are cared for at The Rehabilitation Centre for Children and Adolescents, The Queen Silvia Children's Hospital. At the same hospital, children with BEEC and PUV are cared for in the Urology section in the Pediatric Surgery Department. The team working with children with NBD, BEEC, and PUV includes registered nurses specially trained in urotherapy, pediatric urologists, pediatric nephrologists and pediatric neurologists.

Between 2000 and 2010, 60 individuals (41 boys and 19 girls) were consecutively operated on with reconstructive surgery of the lower urinary tract. The diagnoses in the study were NBD (MMC 31, Anal atresia 8, Other 3), BEEC (13), isolated epispadias (IE) (2) and posterior urethral valves (PUV) (3). Cloacal exstrophy patients were excluded. The procedures performed for each diagnosis are shown in Table 1. Augmentation in NBD was always performed when YD (Young-Dees) or Kropp's procedure [5] was chosen. In children with BEEC and bladder capacity above $80-100 \mathrm{ml}$ at the time of YDL (Young-Dees-Leadbetter, where the bladder neck plasty was combined with neoimplantation of the ureters) [6], augmentation was performed as a second procedure if needed. Further surgical procedures were appendicovesicostomy (Mitrofanoff) [7], Monti procedure [4] when the appendix was not suitable, Sling [8] and Kock nipple [9].

The first follow-up after surgery was performed after three months including the following investigations: cystometry, imaging $\mathrm{Tc} 99^{\mathrm{m}}$-mercapto acetyltriglycine renography (MAG3), renal function with GFR (51-chromium edetic acid), voidingcystourethrography (VCUG), urine culture and measurement of residual urine after CIC. Appropriate laboratory tests (hemoglobin, serum electrolytes, creatinine, bicarbonate) were also obtained. Follow-up thereafter was performed annually according to a structured protocol including the same investigations as at three month after surgery, except MAG3 and GFR which was only performed at ages 6, 12 and 16 years. Instead ultrasonography of upper urinary tract was performed annually and in the blood testing vitamin B12 and folic acid were also included. Cystoscopy was performed 5 years postoperatively, and subsequently every second to third year or when there were difficulties with catheterisation or when hematuria occurred.

The aim with our bladder treatment was to keep a bladder capacity not exceeding bladder capacity according to age and a basal pressure not exceeding $20 \mathrm{~cm} \mathrm{H}_{2} \mathrm{O}$. In addition, daily irrigation of the bladder was always implemented. With time frequency of irrigations were decreased if the mucus production was minimal, but still it was recommended to be done on a regular basis. Another aim was to increase the size of the CIC catheter to make evacuation of urine faster and easier for the patient. At the three-month visit the catheter size was increased from $10 \mathrm{Ch}$ to $12 \mathrm{Ch}$. With time the catheter size was further increased (Ch 14). According to these aims the follow up program for reconstructive surgery patients, focused on how well the patient managed to follow the instructions about timed CIC, regular irrigations of the bladder and to check that the bladder emptying was complete (ultrasonography) and the catheterization technique correct. For support between the visits, the urotherapist could be reached by telephone during all working hours by the patient and their family. Each patient 
Table 1. Primary surgical procedures according to diagnoses. NBD (neurogenic bladder dysfunction), BEEC (bladder exstrophy-epispadias complex), IE (isolated epispadias), YD (Young-Dees), YDL (Young-Dees-Leadbetter), sec proc (second procedure).

\begin{tabular}{|c|c|c|c|}
\hline Diagnosis & NBD (42) No. & BEEC/IE (15) No. & Urethral valves (3) No. \\
\hline Augmentation (45) & 33 & 9 (+3 sec proc) & \\
\hline Mitrofanoff (48) & 31 & 13 (+1 sec proc) & 3 \\
\hline Monti (9) & 9 & & \\
\hline YD (6) & 4 & 2 & \\
\hline YD sling (4) & 4 & & \\
\hline YDL (14) & 3 & 11 & \\
\hline Kropp (10) & 9 (+1 sec proc) & & \\
\hline Isolated sling (3) & 3 & & \\
\hline Bladder neck primary closure (5) & 1 (+2 sec proc) & 2 & \\
\hline
\end{tabular}

had his/her own urotherapist, in order to secure good knowledge about the patient's problem and needs and to make compliance from the patient and family as good as possible.

Complications were consecutively noticed when children were followed-up according to the structured protocol or when medical problems occurred in between. Complications were identified as: bladder stone, rupture of the bladder, obstruction or kinking of the CIC-channel, bowel obstruction, need of re-augmentation, malignancy, rupture or stricture of the BNP and obstruction of the vesicoureteral junction.

\section{Results}

Median age at surgery was 11 years (range 1.3 - 21). The median follow up time after surgery was 7 years $(1$ 10). In total, 27 of 60 (45\%) individuals had complications after reconstructive surgery (16/42 with NBD, 10/15 with BEEC/IE and 1/3 with PUV). Redo surgery due to complications was needed in 16 of the patients (27\%): 7/42 (17\%) with NBD and 7/15 (47\%) with BEEC/IE (Table 2).

\subsection{Augmentation}

Augmentation was performed in 45 patients, with use of ileum in 44 cases and colon in one. All were dependant on CIC for emptying of the augmented bladder. In 3 of 12 augmented BEEC cases (25\%), the procedure was performed as a second step after earlier YDL procedure, due to low-compliant, low-capacity bladders and with urinary leakage in two cases. Overall complications were seen in 7 patients while redo surgery was needed in 5 individuals (11\%) on 6 occasions.

Bladder stones were found in 3 patients (7\%) (Table 2). Stone formation in one of the patients was probably due to the fact that he chose to have an indwelling catheter during school-time to avoid CIC outside home. The two remaining patients had poor CIC and irrigation regimens; one showed improvement in regimen after stone removal and had no stone recurrences during the study, while the other did not have an improvement in regimen and had an additional bladder-stone incidence.

Bladder rupture occurred in 2 patients (Table 2): One boy in a bicycle accident 4 months postoperatively and in the other boy the cause was neglect of CIC-regimen and irrigation.

Detrusor overactivity occurred after augmentation in 2 patients with NBD. After treatment with anticholinergics, continence improved. No re-augmentation was needed.

Malignancy was not seen during the follow up period.

\subsection{Alternative CIC-Channel}

There were 57 channels performed. Complications were noticed in 15 individuals (26\%) (Table 2): angulation 
Table 2. Complications in relations to diagnoses and previous studies. NBD (neurogenic bladder dysfunction), BEEC (bladder exstrophy-epispadias complex), IE (isolated epispadias), PUV (posterior urethral valve) and VU (vesicoureteral).

\begin{tabular}{|c|c|c|c|c|c|}
\hline \multirow{2}{*}{ Complications } & \multicolumn{5}{|c|}{ Patients in need of reoperation, number [\%] } \\
\hline & NBD & $\mathrm{BEEC} / \mathrm{IE}$ & PUV & Total & Previous studies (references) \\
\hline $\begin{array}{l}\text { Augmentation related } \\
\text { - Bladder stones } \\
\text { - } \text { Bladder rupture } \\
\text { - } \text { Re-augmentation } \\
\text { - Malignancy }\end{array}$ & $\begin{array}{c}2 / 33[6] \\
1 / 33[3] \\
0 \\
0\end{array}$ & $\begin{array}{c}1 / 12[8] \\
1 / 12[8] \\
0 \\
0\end{array}$ & & $\begin{array}{c}3 / 45[7] \\
2 / 45[4] \\
0 \\
0\end{array}$ & $\begin{array}{l}6 \%-27 \%[12]-[14] \\
6 \%-14 \%[11][12] \\
0 \%-11 \%[12][16]\end{array}$ \\
\hline 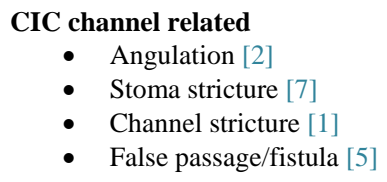 & $\begin{array}{c}3 / 40[7] \\
1 \\
1 \\
1\end{array}$ & $\begin{array}{c}2 / 14[14] \\
2\end{array}$ & $0 / 3$ & $5 / 57[9]$ & $8 \%-39 \%[15]$ \\
\hline $\begin{array}{c}\text { BNP related } \\
\text { - } \quad \text { Rupture } \\
\text { - } \quad \text { Stricture }\end{array}$ & $2 / 24[8]$ & $\begin{array}{l}1 / 15[7] \\
1 / 15[7]\end{array}$ & & 3/39 [8] & \\
\hline VU-junction obstruction & & 2/13 [13] & & & \\
\hline Bowel obstruction & $2 / 42[5]$ & $1 / 14[7]$ & & $3 / 56[5]$ & $3 \%-9 \%[11]$ \\
\hline
\end{tabular}

[2], stricture in stoma [7], stricture in channel [1], false passage [3] and abscess/fistula [2]. No leakage through the channel was observed between the catheterizations, but one patient with a Monti-channel had leakage beside the catheter in the beginning of each emptying. Problems with obstructed stoma and angulation of the channel were seen mostly during the first year postoperatively. In most patients [10] with complications the problem was solved by an indwelling catheter used overnight or for some days to maximum four weeks, whereas in 5 (9\%) a surgical revision was made on 7 occasions (Table 2). The surgically treated complications were angulation with false passage (Mitrofanoff changed to Kock nipple, 1 patient), obstructed channel due to impaired blood supply (Mitrofanoff changed to Monti, 1 patient), stoma granuloma and narrow appendix already from start (stomarevision, 3 patients, 5 procedures).

\subsection{Bladder Neck Plasty}

Sphincter insufficiency with need of BNP was noted in 39 individuals and reoperations were performed in 4 (10\%) (Table 2). In the NBD children two (8\%) had rupture of the BNP: one girl with augmentation and a rectus fascia sling started to leak 3 months postoperatively and a boy had an iatrogenic damage of his BNP caused by a catheter forced through his native urethra during anesthesia in another hospital. In a further case, a girl had an abscess in a SIS sling seroma, which healed without need for re-do surgery.

In the BEEC/IE, BNP was performed in all patients to improve continence: 2 boys had YD without moving the ureters, 11 children a modified YDL and 2 primary bladder neck closure. One boy (7\%) had urinary leakage through his urethra despite YDL and augmentation. He had re-do of his YDL and became dry. One of the two boys (7\%) with YD showed obstruction in his BNP at puberty and was treated with incision. The other boy with YD, performed already at 1.3 years of age, developed urethral stricture below the BNP and was re-operated with inlay of buccal mucosa graft in the posterior urethra with good result. These two boys are the only ones voiding spontaneously without CIC, the older boy had a Mitrofanoff from start, but is not using it.

Obstruction in the vesicoureteral junction was observed in 2 individuals with BEEC surgically treated with YDL. Primary both were treated with endoscopic dilatations and J-stent placement with good result in one but with need of surgical revision in the other.

Bowel obstruction was seen in 3 patients (5\%). The reasons were leakage in the anastomosis of the small intestine [1], kinking and adhesions some days after a laparotomy due to bladder rupture [1] and postoperative 
adhesions at two occasions within 1 year after reconstructive surgery [1].

\section{Discussion}

Overall we found a rate of stone formation and perforation after bladder augmentation that was comparable to the lowest complication rates reported in earlier studies [11]-[14]. Also complications from the CIC channel needing reoperations were in the lower interval as compared with previous reports. We hypothesise that the strict follow-up program with a personal urotherapist appointed for each patient, contribute to the results. This is also in line with the earlier studies in which careful postoperative surveillance [11] [12] and recommendation of daily irrigation of the augmented bladder [13], was considered to contribute to the low complication rate.

However, it is difficult to compare the results between studies, as there is often a mixture of diagnoses, types of reconstructions performed, tissues used and times for follow up. Of further relevance for development of complication is whether the individuals perform CIC through the native urethra or through an alternative channel [11]. In our material, the diagnoses were divided, almost all individuals were augmented with ileum, all but one performed CIC through an alternative channel (Mitrofanoff or Monti) and the median follow up time was 7 years.

A bladder stone formation rate of 7\% in patients with augmentation performing CIC is among the lowest reported [11]-[13]. That careful postoperative surveillance and daily irrigation of the augmented bladder is important was shown by Hensle et al. [14]. They compared 2 groups of bladder-augmented individuals: those who irrigated the bladder daily and those who did not irrigate. The incidence of bladder stones was $7 \%$ and $45 \%$ respectively.

Perforation rate was $4 \%$ in our material. This should be compared to Kispal et al. [12] who showed corresponding incidence in an ileum-augmented group of patients with comparable follow up time and regimen. Metcalfe et al. [11] also had a low incidence (7\%) after a longer follow up duration of 13 years. Complications requiring surgery in the CIC channel were observed in about 9\% of our patients and on par with DeFoor [15]. We did not have any patients in need of re-augmentation [16] or who developed malignancy. Some authors [11] suggest that these situations are seen first after 10 years. The frequency rate of bowel obstruction was similar to other reports (3\% - 8\%) [11].

A limitation with this kind of retrospective study, as the present, is that we do not know for certain the compliance in CIC- and irrigation regimen, even if the intention was close surveillance. Within the first postoperative year some of the patients had an episode of pain from the bladder region. At cystoscopy local inflammation and a lot of mucus in the bottom of the bladder were found. After intensive irrigation with support from the urotherapists and information to the parents that they had to be more involved in the care of the reservoir, the situation was normalized. In the 3 individuals with bladder stones and in 1 of the 2 individuals with perforation there was a well known bad compliance with the CIC- and irrigation regimens despite close surveillance by the urotherapist. Overall, problems with the channel were relatively common, seen in $26 \%$ of the patients in our study, but with different support strategies most of the problems were solved. Only 9\% needed surgical revision, and of these 5 patients, 4 needed surgery despite good regimens (due to ischemia and scar formation). Thus, we are convinced that there are 3 kinds of complications where a meticulous postoperative care seems to be of importance; bladder-stone formation, perforation and the function of the CIC channel.

The paediatric population who were operated on with reconstructive surgery should be treated differently depending on cognitive and physical function and parents' ability. Right from the initial discussions, the surgeon must consider what puberty and independence as an adult would mean for the specific individual in relation to their management of a urinary reservoir [17] [18]. In a previous study, we found that the most challenging period for individuals with MMC was after leaving their parents' home.

In our experience, complication rates will not be reduced only through a combination of visits to the urologist and providing relevant and thorough information to the patient and family regarding CIC-care. To achieve the best results the caregiver needs experience and knowledge about the complex dysfunction seen in individuals with MMC and/or BEEC. Personal knowledge is also needed, as dysfunctions are individually unique. A wellinformed specially trained nurse, the urotherapist, must gain the patient's confidence in order to guide them and argue in favor of the best treatment. One difficulty is that MMC involves impaired prospective memory and capacity for initiative. Therefore, the urotherapist often has to work in close cooperation with the urologist. Thus, one could speculate whether the low complication rates in stone formation, bladder rupture and function of CIC channel is a result of our team composition with a personal urotherapist as the first line of care for children and 
adolescents after reconstructive surgery. In the cases, where complications occurred, it was obvious that the regimens were not performed despite support from the urotherapists. In our opinion, reconstructive surgery in lower urinary tract performed during childhood should only be made at centres which are aware of and capable of handling the postoperative challenges that follow these kinds of complicated procedures.

\section{Conclusion}

Complication rates were among the lowest reported for stones, perforation and reoperations of CIC channels but average for bowel obstruction. Bladder stones and perforation were seen in individuals with bad compliance with recommended CIC- and irrigation regimens. Reoperations after reconstructive surgery in children might be reduced by a meticulous follow-up surveillance. Specially trained nurses, as first line care, could be one way to motivate the patients in the CIC and irrigation regimen.

\section{Acknowledgements}

Special thanks to the urotherapists at the The Rehabilitation Centre for Children and Adolescents, and at the Pediatric Surgery Department at The Queen Silvia Children's Hospital: Birgitta Lindehall, Magdalena VuMinhArnell, Inger Odeholm, Carina Perdsjö, Gloria Davidsson, Anna-Lena Hellström, Sonja Kruse, Monica Berntsson, Monica Dorosckiewicz and Charlotte Arfwidsson.

\section{References}

[1] Bauer, S.B., Reda, E.F., Colodny, A.H. and Retik, A.B. (1986) Detrusor Instability; a Delayed Complication in Association with the Artificial Sphincter. Journal of Urology, 135, 1212-1215.

[2] Stein, R., Schröder, A. and Thuroff, J.W. (2012) Bladder Augmentation and Urinary Diversion in Patients with Neurogenic Bladder: Surgical Considerations. Journal of Pediatric Urology, 8, 153-161. http://dx.doi.org/10.1016/j.jpurol.2011.11.014

[3] Austin, J.C. (2008) Long-Term Risks of Bladder Augmentation in Pediatric Patients. Current Opinion in Urology, 18, 408-412. http://dx.doi.org/10.1097/MOU.0b013e328300587c

[4] Monti, P.R., Lara, R.C., Dutra, M.A. and de Carvalho, J.R. (1997) New Techniques for Construction of Efferent Conduits Based on the Mitrofanoff Principle. Urology, 49, 112-115. http://dx.doi.org/10.1016/S0090-4295(96)00503-1

[5] Kropp, K.A. and Angwafo, F.F. (1986) Urethral Lengthening and Reimplantation for Neurogenic Incontinence in Children. Journal of Urology, 135, 533-536.

[6] Leadbetter Jr., G.W. (1964) Surgical Correction of Total Urinary Incontinence. Journal of Urology, 91, 261-266.

[7] Mitrofanoff, P. (1980) Cystomstomie continente trans-appendiculaire dans le traitement des vessies neurologiques. Chir Pediatr, 21, 297-305.

[8] Barthold, J.S., Rodriguez, E., Freedman, A.L., Fleming, P.A. and Gonzalez, R. (1999) Results of the Rectus Fascial Sling and Wrap Procedures for the Treatment of Neurogenic Sphincteric Incontinence. Journal of Urology, 161, 272-274. http://dx.doi.org/10.1016/S0022-5347(01)62131-1

[9] Kock, N.G., Myrvold, H.E., Nilsson, L.O. and Ahrén, C. (1980) Construction of a Stable Nipple Valve for the Continent Ileostomy. Annales Chirurgiae et Gynaecologiae, 69, 132-143.

[10] Kurzrock, E.A. (2009) Pediatric Enterocystoplasty: Long-Term Complications and Controversies. World Journal of Urology, 27, 69-73. http://dx.doi.org/10.1007/s00345-008-0335-3

[11] Metcalfe, P.D., Cain, M.P., Kaefer, M., Gilley, D.A., Meldrum, K.K., Misseri, R., et al. (2006) What Is the Need for Additional Bladder Surgery after Bladder Augmentation in Childhood? Journal of Urology, 176, 1801-1805. http://dx.doi.org/10.1016/j.juro.2006.03.126

[12] Kispal, Z., Balogh, D., Erdei, O., Kehl, D., Juhasz, Z., Vastyan, A.M., et al. (2011) Complications after Bladder Augmentation or Substitution in Children: A Prospective Study of 86 Patients. BJU International, 108, 282-289. http://dx.doi.org/10.1111/j.1464-410X.2010.09862.x

[13] van den Heijkant, M., Haider, N., Taylor, C. and Subramaniam, R. (2011) Efficacy of Bladder Irrigation and Surveillance Program in Prevention of Urinary Tract Infections and Bladder Calculi in Children with an Ileocystoplasty and Bladder Neck Repair. Pediatric Surgery International, 27, 781-785. http://dx.doi.org/10.1007/s00383-011-2913-5

[14] Hensle, T.W., Bingham, J., Lam, J. and Shabsigh, A. (2004) Preventing Reservoir Calculi after Augmentation Cystoplasty and Continent Urinary Diversion: The Influence of an Irrigation Protocol. BJU International, 93, 585-587.

http://dx.doi.org/10.1111/j.1464-410X.2003.04664.x 
[15] De Foor, W.R., Heshmat, S., Minevich, E., Reddy, P., Koyle, M. and Sheldon, C. (2009) Long-Term Outcomes of the Neobladder in Pediatric Continent Urinary Reconstruction. Journal of Urology, 181, 2689-2693. http://dx.doi.org/10.1016/j.juro.2009.02.039

[16] Vajda, P., Buyukunal, C.S., Soylet, Y., Danismed, N., Juhasz, Z. and Pinter, A.B. (2006) A Therapeutic Method for Failed Bladder Augmentation in Children: Re-Augmentation. BJU International, 97, 816-819. http://dx.doi.org/10.1111/j.1464-410X.2006.06095.x

[17] VuMinh Arnell, M., Seljee Svedberg, K., Lindehall, B., Jodal, U. and Abrahamsson, K. (2012) Adults with Myelomeningocele; an Interview Study about Life Situation and Bladder and Bowel Management. Journal of Pediatric Urology, 9, 267-271.

[18] Woodhouse, C.R.J., Neild, G.H., Yu, R.N. and Bauer, S. (2012) Adult Care of Children from Pediatric Urology. Journal of Urology, 187, 1164-1171. http://dx.doi.org/10.1016/j.juro.2011.12.011 